Supporting Information

\title{
Rational Design of High-Energy-Density Polymer Composites by Machine Learning Approach
}

Ming-Xiao Zhu, ${ }^{*}$ Qiu-Cheng Yu, Heng-Gao Song, Ting-Xin Chen, Ji-Ming Chen

College of New Energy, China University of Petroleum (East China), 266580 Qingdao, China

*Corresponding authors:

(Ming-Xiao Zhu*) E-mail: zhumx@upc.edu.cn 
Table S1 The feature values for different polymers in the fingerprint.

\begin{tabular}{cc}
\hline Polymers & Feature \\
\hline Polypropylene (PP) & 1 \\
Polyvinylidene fluoride (PVDF) & 2 \\
P(VDF-HDP) & 3 \\
P(VDF-TrFE-CFE) & 4 \\
Polyimide (PI) & 5 \\
Polymethyl methacrylate (PMMA) & 6 \\
Divinyltetramethyldisiloxane- & 7 \\
bis(benzocyclobutene) (BCB) & 8 \\
Poly(ether imide) (PEI) & 9 \\
Poly(phthalazinone ether ketone) (PPEK) & 10 \\
Fluorenyl polyester (FPE) & 11 \\
Polycarbonate (PC) & 12 \\
P(VDF-CTFE) & 13 \\
P(VDF-HDP)+PMMA & 14 \\
Polyacrylonitrile (PAN) & 15 \\
Polyethylene (PE) & 16 \\
P(VDF-TrFE-CFE)+PMMA &
\end{tabular}

Table S2 The electrical properties of different nanofillers in the fingerprint.

\begin{tabular}{cccc}
\hline Nanofillers & permittivity & $\begin{array}{c}\text { Electrical } \\
\text { conductivity }\end{array}$ & Bandgap \\
\hline $\mathrm{Al}_{2} \mathrm{O}_{3}$ & 9.4 & $1 \mathrm{e}-12$ & 9.5 \\
$\mathrm{HfO}_{2}$ & 25 & $1 \mathrm{e}-11$ & 5.8 \\
$\mathrm{TiO}_{2}$ & 110 & $1 \mathrm{e}-10$ & 3.5 \\
$\mathrm{BNNS}_{\mathrm{MgO}}$ & 4 & $1 \mathrm{e}-11$ & 5.97 \\
$\mathrm{BaTiO}_{3}$ & 9.7 & $5 \mathrm{e}-15$ & 7.8 \\
$\mathrm{ZnO}$ & 1000 & $8 \mathrm{e}-8$ & 3 \\
$\mathrm{SrTiO}_{3}$ & 10 & $1 \mathrm{e}-12$ & 3.37 \\
$\mathrm{BZCT}$ & 200 & $1 \mathrm{e}-9$ & 3.4 \\
$\mathrm{BaSrTiO}_{3}$ & 400 & $5 \mathrm{e}-7$ & 3 \\
$\mathrm{Bi}_{2} \mathrm{~S}_{3}$ & 300 & $1 \mathrm{e}-9$ & 3 \\
$\mathrm{SiO}_{2}$ & 2000 & $1 \mathrm{e} 3$ & 0.5 \\
$\mathrm{Ni}_{2}\left(\mathrm{OH}_{2}\right.$ & 4 & $1 \mathrm{e}-13$ & 9 \\
$\mathrm{NaNbO}_{3}$ & 100 & $1 \mathrm{e}-4$ & 5.5 \\
& 200 & $1 \mathrm{e}-9$ & 3.4 \\
\hline
\end{tabular}




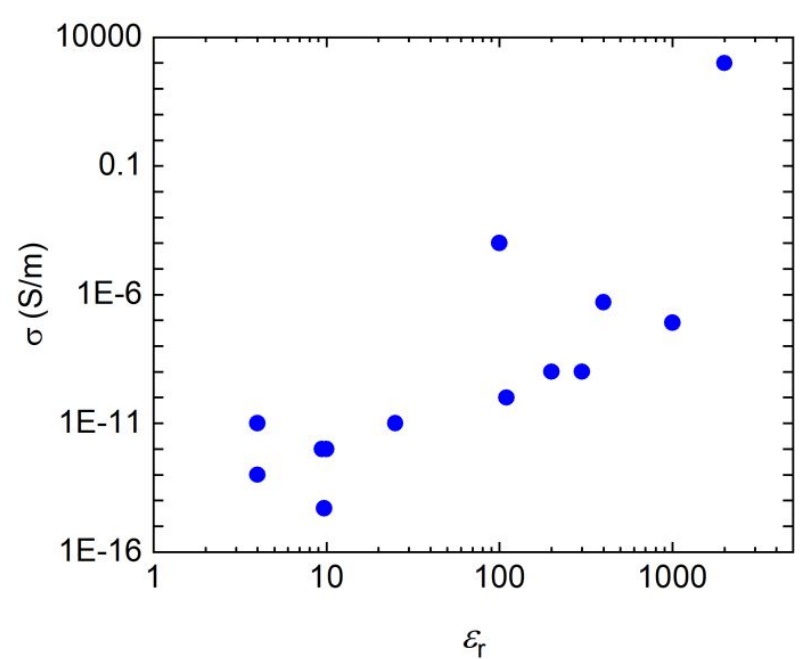

(a)

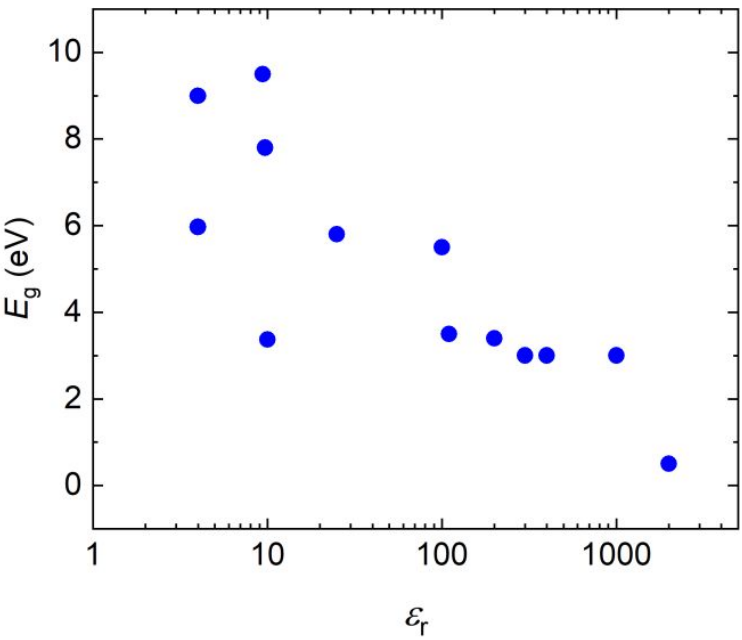

(b)

Fig. S1 The relationships between dielectric parameters of various inorganic nanofillers. (a) Relationship between permittivity and electrical conductivity. (b) Relationship between permittivity and bandgap. 

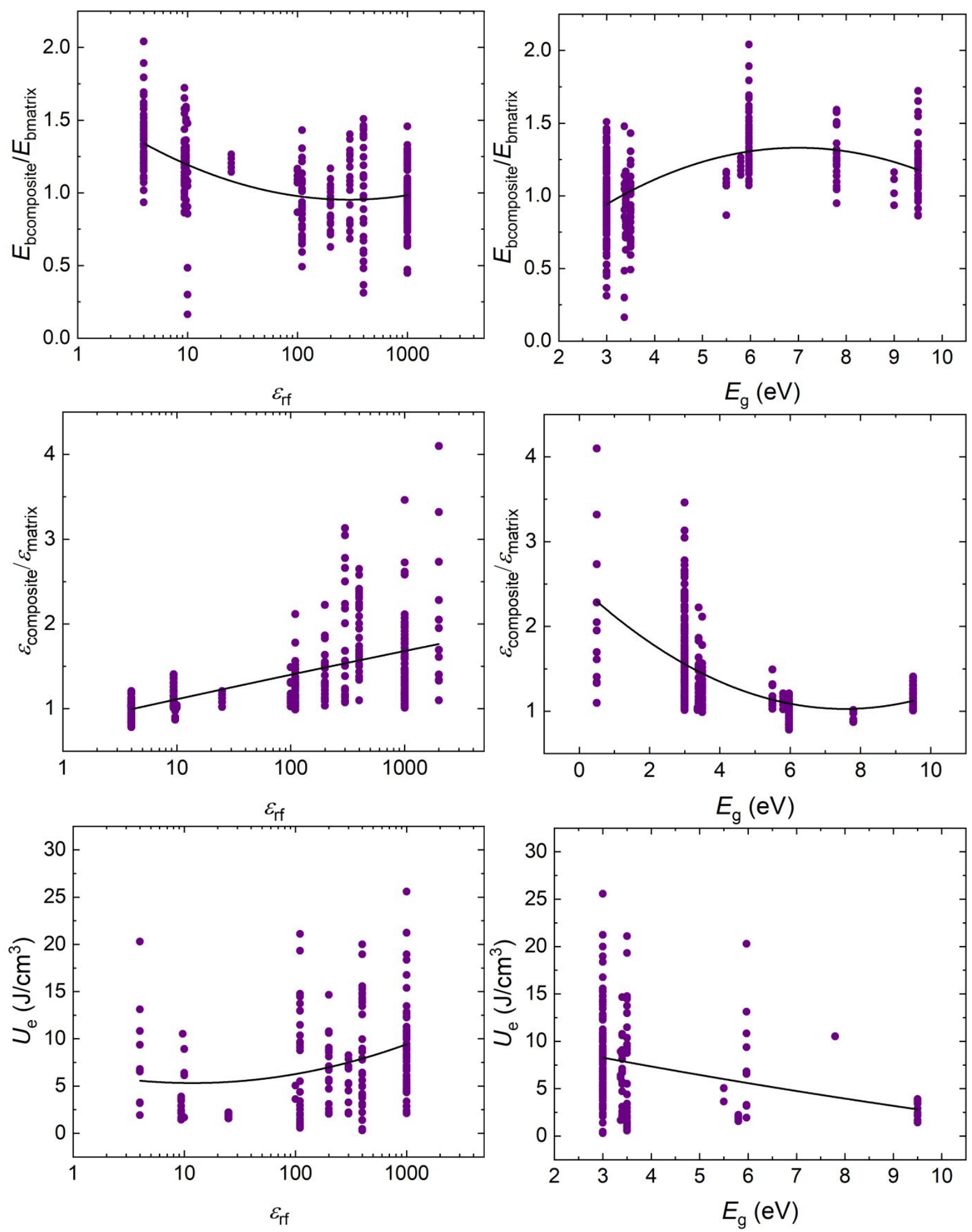

Fig. S2 The dependence of $E_{\mathrm{b}}, \varepsilon_{\mathrm{r}}$ and $U_{\mathrm{e}}$ on $\varepsilon_{\mathrm{rf}}$ and $E_{\mathrm{g}}$ of nanofillers. 

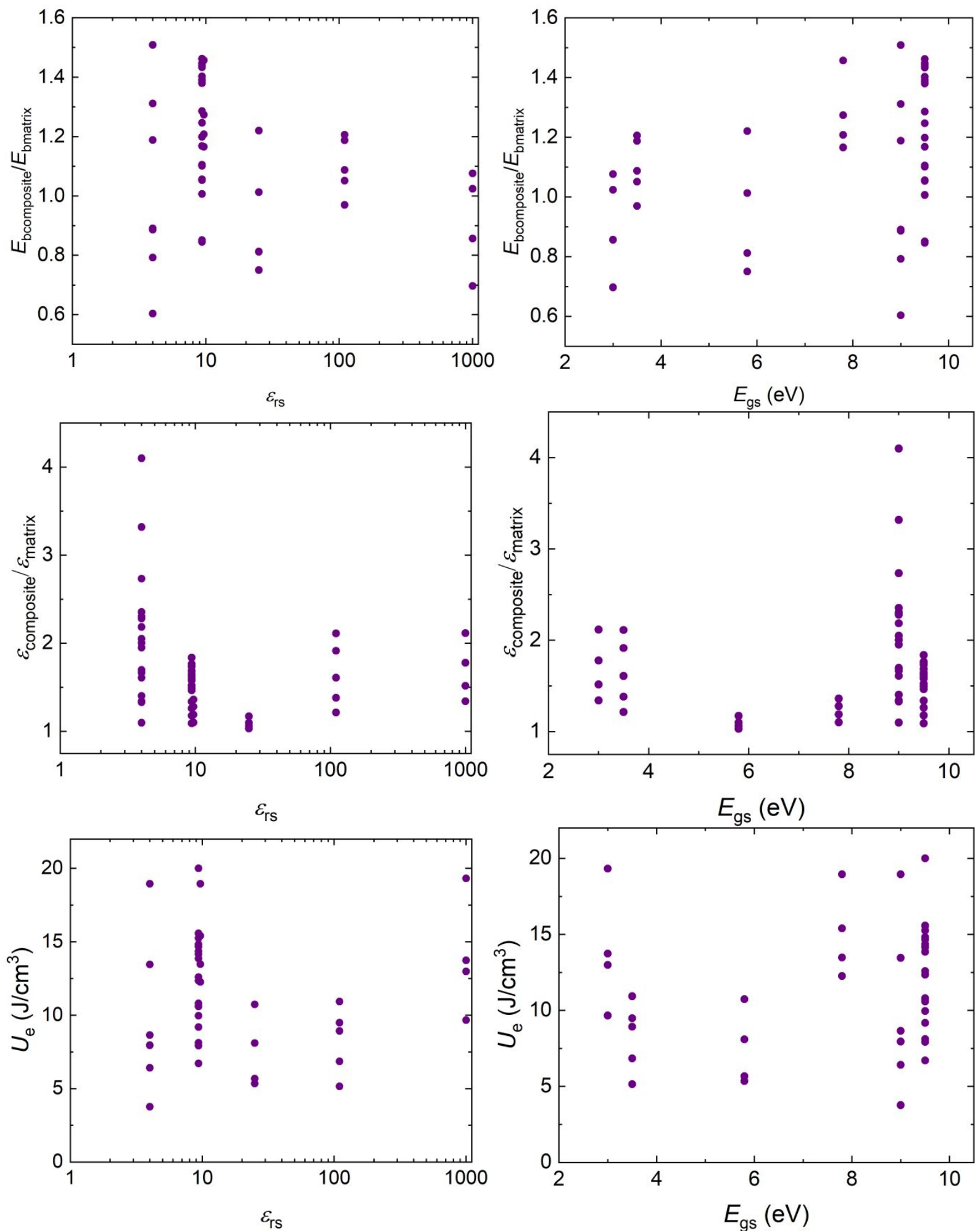

Fig. S3 The dependence of $E_{\mathrm{b}}, \varepsilon_{\mathrm{r}}$ and $U_{\mathrm{e}}$ on $\varepsilon_{\mathrm{rs}}$ and $E_{\mathrm{gs}}$ of nanofillers shell layer. 


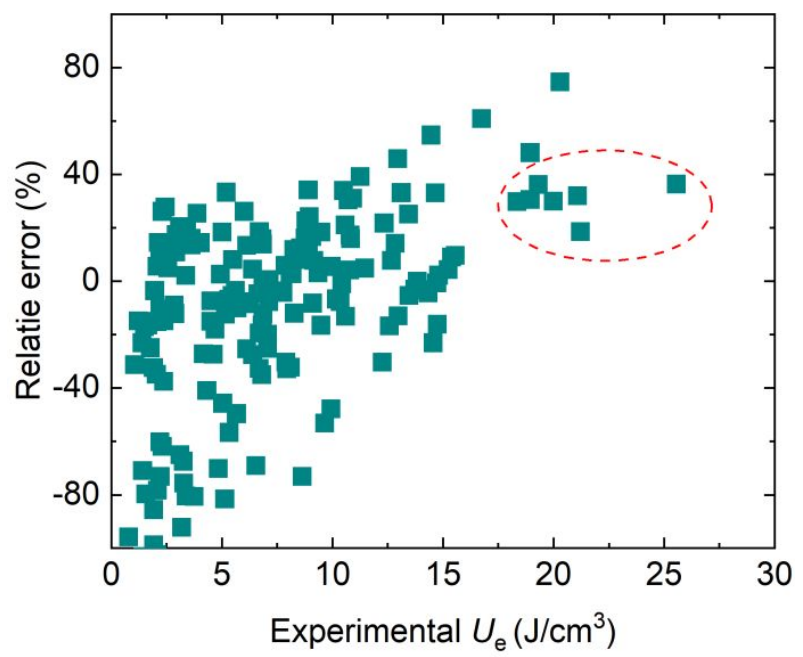

Fig. S4 The distribution of prediction relative error for $U_{\mathrm{e}}$. 

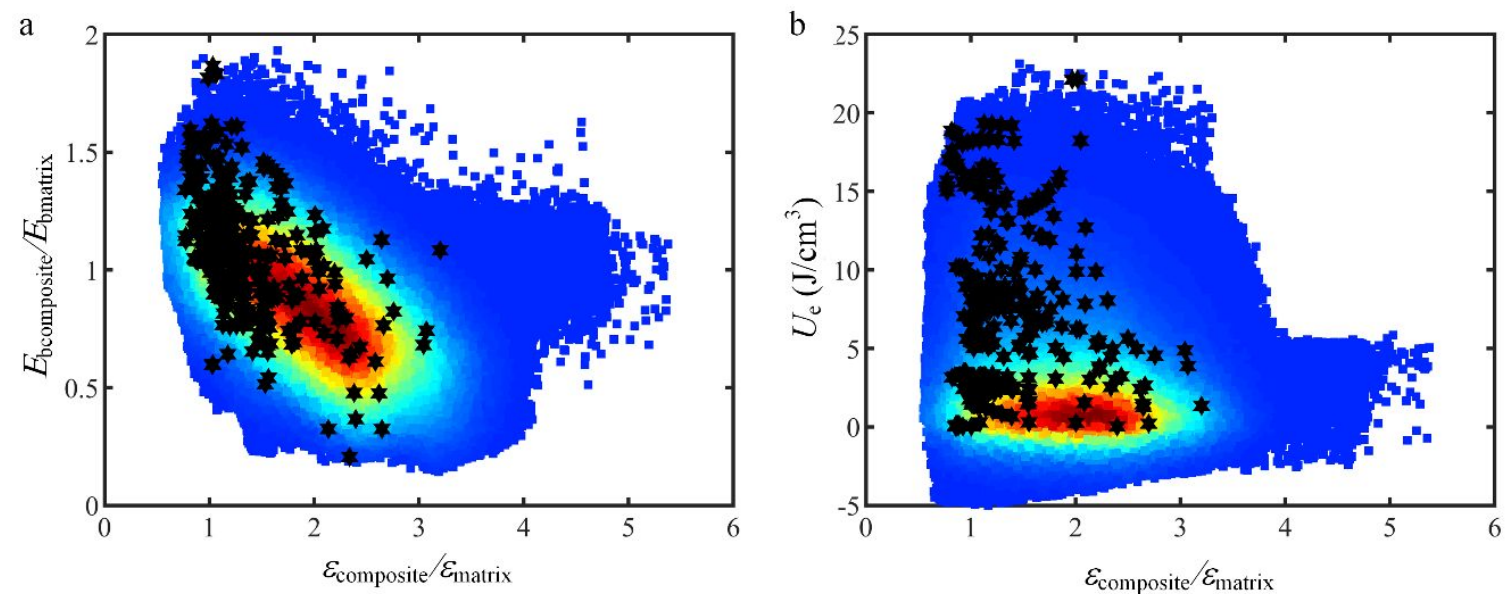

Fig. S5 The distributions of predicted $\varepsilon_{\mathrm{r}}, E_{\mathrm{b}}$ and $U_{\mathrm{e}}$ in one ML test.

Table S3 The nanofillers shape and geometric distributions parameters of optimized doping scheme.

\begin{tabular}{cccccc}
\hline $\begin{array}{c}\text { Fillers } \\
\text { distribution }\end{array}$ & Polymer & Vol $\%$ & $l_{\mathrm{a}}(\mathrm{nm})$ & $l_{\mathrm{b}} / l_{\mathrm{a}}$ & $l_{\mathrm{c}} / l_{\mathrm{a}}$ \\
\hline B1 & PVDF & 7.25 & 3.5 & 150 & 150 \\
B2 & PVDF/PP & 7.25 & 5.5 & 200 & 200 \\
B3 & PP/PVDF & 7.75 & 5 & 1 & $200 / 1000$ \\
P1 & PP & - & 150 & 1 & 1 \\
P2 & PP & - & $3 \sim 200$ & $1.1 \sim 40$ & $1.1 \sim 40$ \\
P3 & PP & - & $4 \sim 200$ & 1 & $1.1 \sim 40$ \\
U1 & P(VDF-HFP) & - & 100 & 200 & 200 \\
U2 & P(VDF-HFP) & - & 100 & 1 & $25 / 200$ \\
U3 & P(VDF-HFP) & - & $100 \sim 200$ & 15 & 15 \\
\hline
\end{tabular}




\section{Note S1: Gaussian Process Regression}

For Gaussian process regression, the objective values, $y=\left[y_{1}, y_{2}, \ldots, y_{N}\right]^{\mathrm{T}}$, are assumed to conform a multivariate Gaussian distribution $[1,2]$ :

$$
\boldsymbol{y} \sim N(\boldsymbol{0}, \boldsymbol{K})
$$

The mean value is often assumed to be zero. $\boldsymbol{K}$ is the covariance matrix, whose elements are determined with a kernel function $k\left(x_{1}, x_{2}\right)$. Kernel functions and their hyperparameters have a great influence on the prediction accuracy, which are optimized during the model training process with the goal of maximizing the log-likelihood estimate. The objective value $f\left(x^{*}\right)$ of a test data $\boldsymbol{x}^{*}$ can be determined from the estimated Gaussian distribution. 


\section{Note S2: Phase-Field Model}

In the phase-field model, the damage status of material is represented with a continuous order variable $\eta(\boldsymbol{r}, t)$, which is both spatially and temporally dependent, where $t$ is time and $\boldsymbol{r}$ is the spatial position. The $\eta(\boldsymbol{r}, t)$ varies from intact status, $\eta(\boldsymbol{r}, t)=0$, to the damaged status, $\eta(\boldsymbol{r}, t)=1$. The relative permittivity and electrical conductivity of the damaged phase $\varepsilon_{\mathrm{B}}$, and $\sigma_{\mathrm{B}}$ are set as a high value of $10^{4}$ and $10^{-5}$ to represent the electrical tree channels. The permittivity and electrical conductivity of the composite during the microstructure evolution is dependent on $\eta(\boldsymbol{r}, t)$, and is interpolated by [3]

$$
\begin{aligned}
& \varepsilon_{\mathrm{r}}(\boldsymbol{r})=\left(4 \eta(\boldsymbol{r})^{3}-3 \eta(\boldsymbol{r})^{4}\right) \varepsilon_{\mathrm{B}}+\left[1-\left(4 \eta(\boldsymbol{r})^{3}-3 \eta(\boldsymbol{r})^{4}\right)\right] \varepsilon_{\mathrm{C}}(\boldsymbol{r}) \\
& \sigma(\boldsymbol{r})=\left(4 \eta(\boldsymbol{r})^{3}-3 \eta(\boldsymbol{r})^{4}\right) \sigma_{\mathrm{B}}+\left[1-\left(4 \eta(\boldsymbol{r})^{3}-3 \eta(\boldsymbol{r})^{4}\right)\right] \sigma_{\mathrm{C}}(\boldsymbol{r})
\end{aligned}
$$

where, $\varepsilon_{\mathrm{C}}(\boldsymbol{r})$ and $\sigma_{\mathrm{C}}(\boldsymbol{r})$ are the spatially dependent permittivity and electrical conductivity of nanocomposite which are determined by the properties of nanofiller and matrix.

The electrical tree growth is driven by free energy in the system $[4,5]$ :

$$
F=\int_{\Omega}\left[f_{\text {sep }}(\eta(\boldsymbol{r}))+\frac{1}{2} \gamma|\nabla \eta(\boldsymbol{r})|^{2}+f_{\text {ele }}(\eta(\boldsymbol{r}))+f_{\text {joule }}(\eta(\boldsymbol{r}))\right] \mathrm{d} V
$$

where, $f_{\text {sep }}(\eta(\boldsymbol{r}))=\alpha \eta^{2}(1-\eta)^{2}$ is the free energy density that drives the phase separation with $\alpha$ defining the energy barrier between intact and damage status. The second term is the gradient energy density in which $\gamma$ is the gradient energy coefficient. $f_{\text {ele }}(\eta(\boldsymbol{r}))=-1 / 2 \varepsilon(\boldsymbol{r}) \boldsymbol{E}(\boldsymbol{r}) \cdot \boldsymbol{E}(\boldsymbol{r})$ is the electrostatic energy density with $\varepsilon(\boldsymbol{r})$ is the spatially and temporally dependent dielectric permittivity. $f_{\text {joule }}(\eta(\boldsymbol{r}))$ is the thermal energy density with the form of:

$$
f_{\text {joule }}(\eta(\boldsymbol{r}))=-\sigma(\boldsymbol{r}) \boldsymbol{E}(\boldsymbol{r}) \cdot \boldsymbol{E}(\boldsymbol{r}) d t
$$


The local electric field $\boldsymbol{E}(\boldsymbol{r})$ can be obtained by solving the possion equation with spectral iterative perturbation method [6]. The iterative form of depolarization potential $\varphi(\boldsymbol{r})$ is described as follows.

$$
\sigma_{\text {ref }} \sum_{j=1}^{D} \frac{\partial^{2} \varphi(\boldsymbol{r})}{\partial x_{j}^{2}}=\sum_{j=1}^{D} \frac{\partial}{\partial x_{j}}\left[\Delta \sigma(\boldsymbol{r})\left(E_{j}^{\mathrm{ext}}-\frac{\partial \varphi(\boldsymbol{r})}{\partial x_{j}}\right)\right]
$$

where, $\sigma_{\text {ref }}$ is a homogeneous reference of the electrical conductivity, and $\Delta \sigma(\boldsymbol{r})=\sigma(\boldsymbol{r})-\sigma_{\text {ref }}$ is the inhomogeneous perturbation. $D$ is the dimension of space, and $x_{j}$ is $j$ th component of the position vector. $\varphi(\boldsymbol{r})$ is the depolarization potential. $E_{j}^{\mathrm{ext}}$ is external electrical field in the direction of $x_{j}$ axis. The $\varphi(\boldsymbol{r})$ can be obtained by iteratively solve Equation (7) in Fourier space, then the electric field can be calculated as:

$$
E_{j}(\boldsymbol{r})=E_{j}^{\mathrm{ext}}-\frac{\partial \varphi(\boldsymbol{r})}{\partial x_{j}}
$$

where $E_{j}(\boldsymbol{r})$ is electrical field in the direction of $x_{j}$ axis.

The evolution of damage phase is described with a modified Allen-Cahn equation,

$$
\frac{\partial \eta(\boldsymbol{r}, t)}{\partial t}=-L_{0} H\left(f_{\text {ele }}+f_{\text {joule }}-f_{\text {th }}\right)\left[\frac{\partial f_{\text {sep }}(\eta)}{\partial \eta(\boldsymbol{r}, t)}-\gamma \nabla^{2} \eta(\boldsymbol{r}, t)+\frac{\partial f_{\text {ele }}(\eta)}{\partial \eta(\boldsymbol{r}, t)}+\frac{\partial f_{\text {joule }}(\eta)}{\partial \eta(\boldsymbol{r}, t)}\right]
$$

where, $L_{0}$ is kinetic coefficient which represents the speed of damage evolution, $H\left(f_{\text {ele }}-f_{\text {th }}\right)$ is the Heaviside unit step function, $f_{\text {th }}$ is inception energy density to cause insulation damage. The driving forces from the phase separation and electrostatic energy can be derived as follows.

$$
\begin{gathered}
\frac{\partial f_{\text {sep }}(\eta)}{\partial \eta(\boldsymbol{r}, t)}=2 \alpha \eta(1-\eta)(1-2 \eta) \\
\frac{\partial f_{\text {ele }}(\eta)}{\partial \eta(\boldsymbol{r}, t)}=-6\left(\eta^{2}-\eta^{3}\right)\left(\varepsilon_{\mathrm{B}}-\varepsilon_{\mathrm{C}}\right) \varepsilon_{0} \boldsymbol{E}(\boldsymbol{r}) \cdot \boldsymbol{E}(\boldsymbol{r})
\end{gathered}
$$




$$
\frac{\partial f_{\text {joule }}(\eta)}{\partial \eta(\boldsymbol{r}, t)}=-12\left(\eta^{2}-\eta^{3}\right)\left(\sigma_{\mathrm{B}}-\sigma_{\mathrm{C}}\right) \boldsymbol{E}(\boldsymbol{r}) \cdot \boldsymbol{E}(\boldsymbol{r}) d t
$$

A Fourier-space based method can be used to solve the Allen-Cahn equation, which have been illustrated in [4]. Since the $\eta(r, t)$ is rapidly varying for different positions, the gradient energy term is solved with an isotropic form of the Laplacian operator which can be found in [7].

A mesh grid of $N_{x} \Delta x \times N_{y} \Delta y \times N_{z} \Delta z$ with grid size of $\Delta x=\Delta y=\Delta z=20 \mathrm{~nm}$ and $N_{x}=128$, $N_{y}=128, N_{z}=256$ is employed in the simulations. A time step $\Delta t=0.01 \mathrm{~s}$ is used to numerically solve (9). The gradient energy coefficient $\gamma$ is set as $10^{-10} \mathrm{~J} / \mathrm{m}[4]$, and $L_{0}$ is set as $10^{-9}$. The different material properties of matrix, nanofillers even interfacial regions are characterized by $\varepsilon(\boldsymbol{r}, t), \sigma(\boldsymbol{r}, t)$ and $f_{\mathrm{th}}(\boldsymbol{r})$. The critical energy of each component is calculated as $f_{\text {th }}=0.5 \varepsilon_{0} \varepsilon_{\mathrm{r}} E_{\mathrm{b}}^{2}+\sigma E_{\mathrm{b}}^{2} d t$. PVDF is used as the matrix in the simulation, with $\varepsilon_{\mathrm{r}}$ of $10, \sigma$ of $2.12 \times 10^{-12}$, and $E_{\mathrm{b}}$ of $350 \mathrm{kV} / \mathrm{mm}$. The $\varepsilon_{\mathrm{r}}$ and $\sigma$ of nanofillers and shell layers are from Table S2. The coefficient $\alpha$ is set as $10^{8} \mathrm{~J} / \mathrm{m}^{3}$, and a fixed temperature of $300 \mathrm{~K}$ is used in the simulation. To simulate the experimental conditions, a ramp DC electric field with ramping rate of $1 \mathrm{kV} /(\mathrm{mm} \cdot \mathrm{s})$ is applied to the composites. The iterative solution of Poisson's equation is the most time-consuming step in the whole simulation. To speed up the simulation, the initial electric field distribution for iteration is set as that of the latest step of Equation (9).

For calculating the effective permittivity of nanocomposites, the local electric field $\boldsymbol{E}(\boldsymbol{r})$ under alternative voltage is firstly determined by solving the Poisson's equation with spectral iterative perturbation method. The iterative form of depolarization potential $\varphi(\boldsymbol{r})$ is described as follows:

$$
\varepsilon_{\text {ref }} \sum_{j=1}^{D} \frac{\partial^{2} \varphi^{i+1}(\boldsymbol{r})}{\partial x_{j}^{2}}=\sum_{j=1}^{D} \frac{\partial}{\partial x_{j}}\left[\Delta \varepsilon(\boldsymbol{r})\left(E_{j}^{\mathrm{ext}}-\frac{\partial \varphi^{i}(\boldsymbol{r})}{\partial x_{j}}\right)\right]-\frac{\rho(\boldsymbol{r})}{\varepsilon_{0}}
$$


where, $\varepsilon_{\text {ref }}$ is a homogeneous reference of the relative dielectric permittivity, and $\Delta \varepsilon(\boldsymbol{r})=\varepsilon_{\mathrm{r}}(\boldsymbol{r})-\varepsilon_{\text {ref }}$ is the inhomogeneous perturbation. Then the electric filed is calculated by equation (8). After that, the effective permittivity of nanocomposites is determined with the procedures in [8]. 


\section{References:}

[1] J. Schmidt, M. R. G. Marques, S. Botti, M. A. L. Marques, Recent advances and applications of machine learning in solid state materials science, npj Comput. Mater. 5 (2019) 83.

[2] J. Quiñonero-Candela, C. E. Rasmussen, A unifying view of sparse approximate Gaussian process regression, J. Mach. Learn. Res. 6 (2005) 1939-1959.

[3] A. Karma, D.A. Kessler, H. Levine, Phase-Field Model of Mode III Dynamic Fracture, Phys. Rev. Lett., 87 (2001) 045501.

[4] Z.H. Shen, J.J. Wang, Y.H. Lin, C.W. Nan, L.Q. Chen, Y. Shen, High-Throughput Phase-Field Design of High-Energy-Density Polymer Nanocomposites, Adv. Mater., $30(2018) 1704380$.

[5] Z. Cai, X. Wang, L. Li, W. Hong, Electrical treeing: A phase-field model, Extreme Mechanics Letters, 28 (2019) 87-95.

[6] J.J. Wang, X.Q. Ma, Q. Li, J. Britson, L.Q. Chen, Phase Transitions and Domain Structures of Ferroelectric Nanoparticles: Phase Field Model Incorporating Strong Elastic and Dielectric Inhomogeneity, Acta Mater. 61 (2013) 7591-7603.

[7] N. Provatas, K. Elder, Phase-Field Methods in Materials Science and Engineering, Weinheim, Wiley-VCH (2010) 262-263.

[8] S.L. Zhong, Z.M. Dang, J.W. Zha, Prediction on Effective Permittivity of 0-3 Connectivity Particle/Polymer Composites at Low Concentration with Finite Element Method, IEEE Trans. Dielectr. Electr. Insul. 25 (2018) 2122-2128. 